\title{
GEO
}

Geograficando

ISSN: 2346-898X

geograficando@fahce.unlp.edu.ar

Universidad Nacional de La Plata

Argentina

\section{Influencia de ENOS en la hidrometeorología de la cuenca alta del río Neuquén}

\author{
Groch, Damián; Cogliati, Marisa Gloria; Finessi, Florencia Gisella \\ Influencia de ENOS en la hidrometeorología de la cuenca alta del río Neuquén \\ Geograficando, vol. 16, núm. 1, 2020 \\ Universidad Nacional de La Plata, Argentina \\ DOI: https://doi.org/10.24215/2346898Xe066
}

Atribución no comercial compartir igual (CC BY-NC-SA) 4.0 


\section{Influencia de ENOS en la hidrometeorología de la cuenca alta del río Neuquén}

Influence of ENSO on the upper basin of Neuquén river hydrometeorology

Damián Groch

Departamento de Geografia, Facultad de Humanidades, Universidad Nacional del Comabue, Argentina

Marisa Gloria Cogliati

Departamento de Geografía, Facultad de Humanidades, Universidad Nacional del Comahue, Argentina

Florencia Gisella Finessi

Departamento de Geografía, Facultad de Humanidades, Universidad Nacional del Comahue, Argentina

DOI: https://doi.org/10.24215/2346898Xe066

Recepción: 07 Agosto 2019 Aprobación: 22 Noviembre 2019

Recepción: 07 Agosto 2019

Aprobación: 22 Noviembre 2019

\section{RESUMEN:}

La interacción océano-atmósfera actúa como forzante de los sistemas hidrológicos en diferentes escalas temporales, siendo El Niño-Oscilación Sur (ENOS) uno de ellos. Este trabajo presenta el análisis de variables hidrometeorológicas y su asociación con el Índice Oceánico del Niño (ONI), representativo de ENOS, en la Cuenca Alta del Río Neuquén para el período 2000-2014. Se ha detectado una débil correlación ( $r$ de 0,35). La precipitación, la temperatura, el caudal, el Equivalente de Agua en Nieve y el Área Cubierta de Nieve (ACN) presentaron una débil asociación con el ONI, con un coeficiente de correlación medio de 0,35 y un nivel de significación del 5\% en la Cuenca Alta del Río Neuquén (CARN) para el período 2000-2014. El ACN presentó una correlación muy débil, entre -0,07 para El Niño y 0,14 para La Niña, mientras que las correlaciones entre el caudal y el ONI fueron de 0,51 para la totalidad del período y 0,31 para las fases de El Niño al $5 \%$ de significación, sin ser significativas para períodos de La Niña Palabras clave: El Niño-Oscilación del Sur, Índice Oceánico del Niño, Terra-MODIS, Caudal, Precipitación.

\section{Abstract:}

The ocean-atmosphere interaction acts as a force for hydrological systems at different time scales, El Niño-Southern Oscillation (ENSO) being one of them. This paper presents the analysis of hydrometeorological variables and their association with the Oceanic Niño Index (ONI), representative of ENSO, in the Upper Basin of Neuquén River (UBNR) for the period 2000-2014; detecting a weak correlation ( $r$ of 0,35$)$. Precipitation, temperature and SWE presented weak and moderate associations, with a $r$ lower than $0.44,0.23$ and 0.36 respectively, without a defined behavior for the different phases of ENSO; while the SCA presented a very weak correlation, between -0.07 and 0.14 . Correlation between the flow and the ONI was 0.51 for the entire period and 0,31 for the El Niño phases with a $5 \%$ of significance, without being significant for La Niña periods.

KEYwORDS: El Niño-Southern Oscillation, Oceanic Niño Index, Terra-MODIS, Flow rate, Precipitation.

\section{INTRODUCCIÓN}

Las variaciones climáticas y oceánicas de gran escala se interrelacionan con diversas variables hidrológicas en diferentes partes del planeta, influyendo sobre la vida del hombre y su disponibilidad de recursos, por lo que su estudio permite comprender las dinámicas particulares del sistema y actuar en consecuencia.

El Niño - Oscilación del Sur (ENOS) es el modo dominante de la variabilidad acoplada océano-atmósfera en la escala interanual de diferentes regiones del mundo (Trenberth \& Stepaniak, 2001). El fenómeno ENOS es una perturbación interanual del sistema climático caracterizado por un debilitamiento aperiódico de los vientos alisios y el calentamiento de las capas superficiales en el océano Pacífico Ecuatorial, el cual se manifiesta 
principalmente en regiones costeras del océano Pacífico tropical, con una duración de varios meses, una recurrencia irregular y efectos que varían según su intensidad (Gómez Cole, 2010; Araya Ojeda e Isla, 2016). Esta interacción océano-atmósfera repercute en los sistemas hidrológicos en diferentes escalas temporales, por lo que puede influir en el comportamiento de diversas variables meteorológicas e hidrográficas, como caudal, precipitación y temperatura del aire. El efecto de algunas de ellas, entre muchas otras, fue estudiado por Ropelewski \& Halpert (1989, 1996) y Ropelewski \& Jones (1987). García (1994) describe su influencia para caracterizar la climatología de América del Sur, como también para esclarecer la relación existente con el fenómeno de ENOS.

El estudio del fenómeno ENOS ha aumentado, sobre todo en las últimas décadas, debido a la ocurrencia de eventos naturales extremos que ponen en riesgo a las poblaciones, como sequías e inundaciones con un impacto social y económico relevante en distintas zonas del país y del mundo (CIMOP, 2009). En Argentina, la asociación de ENOS con las inundaciones fue estudiada por Callau Poduje \& Seoane (2013), y con las precipitaciones por Daniels y Veblen (2000), Ribera \& Penalba (2015) y Penalba \& Ribera (2016). Los estudios específicos de ENOS se encuentran poco desarrollados para los Andes Nordpatagónicos, lo que está asociado con la limitada disponibilidad de los datos para el análisis del comportamiento de las precipitaciones, los caudales y la cubierta de nieve en la región.

En la región de los Andes Centrales de Argentina y Chile, Cerveny, Skeeter \& Dewey (1987), Escobar y Aceituno (1998), Gómez Cole (2010), Masiokas, Villalba, Luckman, Le Quesne \& Aravena (2006) y Masiokas et al. (2012) estudiaron el ENOS mediante la correlación entre datos nivológicos, el Índice de Oscilación Sur (SOI, Southern Oscilation Index) y el Índice Oceánico de El Niño (ONI, Oceanic Niño Index). Norte, Simonelli y Heredia (1998) consideraron la precipitación y el caudal de 1957-1997 en la provincia de Mendoza (Argentina) para el establecimiento de asociaciones con ENOS, al igual que Araya Ojeda e Isla (2016) para la segunda mitad del siglo XX en la región de Biobío (Chile). Por otro lado, Martínez, Fernández y Rubio (2012) realizaron dichas asociaciones utilizando datos de caudal de 1950-2000 del río Aconcagua (Chile).

Masiokas, Villalba, Luckman, Le Quesne \& Aravena (2006) y Lauro, Vich y Moreiras (2016) postularon que el caudal representa la función de respuesta o síntesis de todos los procesos que se verifican en una cuenca; y en la región andina -entre los $30^{\circ}$ y $37^{\circ}$ Sur- está estrechamente relacionada con las precipitaciones durante el período invernal y las variaciones de la temperatura durante la marcha del ciclo anual o componente estacional. En dicha región, para el período 1931-2011 la variabilidad interanual de los ríos es significativa y presenta diferentes grados de correlación en función de las fases de ENOS (Aceituno, 1990; Compagnucci \& Vargas, 1998). Las fases cálidas (El Niño) se asocian a precipitaciones níveas por encima de la media y aumento del escurrimiento durante el período estival, mientras que en las fases frías (La Niña) las precipitaciones y el escurrimiento se comportan inversamente (Lauro, Vich y Moreiras, 2016).

Compagnucci y Araneo $(2005,2007)$ plantean que se desconoce aún la relación entre la variabilidad de los caudales y las características de los forzantes climáticos que los determinan en los ríos patagónicos. Los autores añaden que, si bien en los Andes centrales (al norte del río Colorado) las fluctuaciones en escala interanual a decádica de los caudales son reflejo de las variaciones de la Temperatura de Superficie del Mar (TSM) y de la circulación atmosférica, durante el período de invierno y principios de primavera dicha estructura en el norte de la Patagonia se encuentra ausente, lo que insinúa una escasa o baja relación con ENOS.

En la Cuenca Alta del río Neuquén (CARN), estos efectos se asocian con inundaciones, sequías y grandes nevadas en la cuenca, que redundan en problemáticas socioeconómicas y daños materiales, como cortes del suministro eléctrico y de gas, cortes de ruta por la acumulación de nieve, congelamiento de vertientes y arroyos que proveen de agua a la población, mortandad de animales por falta de pasturas e incendios de pastizales asociados a sequias. Por ello, Finessi y Groch (2018) realizaron una primera aproximación al análisis de la variabilidad hidrológica existente y su asociación con fenómenos hidrometeorológicos, considerando el SOI y ONI en la Cuenca Alta del Río Neuquén (CARN). 
Las condiciones hidrometeorológicas de la cuenca adquieren una especial significación al exponer a diferentes riesgos a los centros poblados, generando a su vez problemáticas socio-naturales que impiden el desarrollo de las actividades socio-económicas de la cuenca. Para ello se planteó el diagnóstico de la asociación de diferentes elementos climáticos (precipitación, temperatura), hidrográficos (caudal) y nivológicos (área cubierta de nieve, equivalente en agua de nieve) con el fenómeno ENOS en la CARN.

En este trabajo se presenta el análisis de la relación del fenómeno ENOS con la precipitación, temperatura, caudal, Área Cubierta de Nieve (ACN) y Equivalente de Agua en Nieve (EAN) en la Cuenca Alta del Río Neuquén.

\section{ÁREA DE ESTUDio}

La CARN posee una superficie de $8.282,0 \mathrm{~km}^{2}$ y una marcada amplitud altimétrica que oscila entre los 874,0 y 4.707,0 m s.n.m. Se localiza parcialmente en los departamentos Minas, Chos Malal y Norquín, en el extremo noroeste de la provincia del Neuquén, entre los $36^{\circ} 10^{\prime}-37^{\circ} 47^{\prime} S$ y los $71^{\circ} 13^{\prime}-70^{\circ} 27^{\prime} \mathrm{O}$ (Figura 1). Se encuentra delimitada por la Cordillera del Viento al este, la cual posee una disposición Norte-Sur de aproximadamente 120,0 km de longitud; la Cordillera de los Andes en el sector oeste; mientras que al sur limita con la Sierra Trolón y la Cordillera de Mandolegüe. La cota máxima se localiza al norte de la Cordillera del Viento, en el Vn. Domuyo, mientras que la cota inferior corresponde al cauce del río Neuquén en el punto de cierre de la cuenca. Este se consideró en la estación hidrometeorológica Rahueco, ubicada 16,0 km aguas arriba de la ciudad de Chos Malal (mayor centro poblado de la región). 


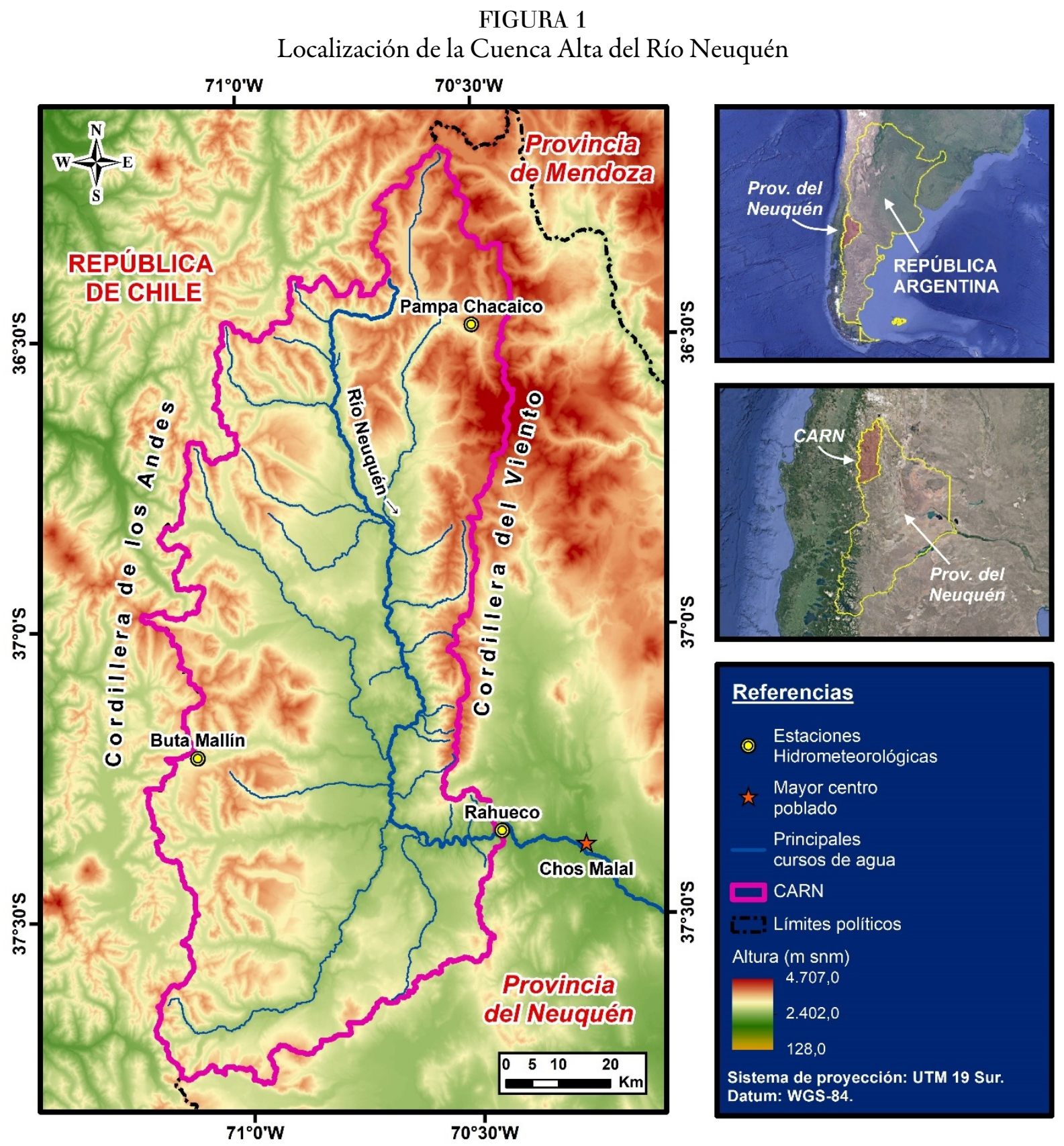

Fuente: Elaboración propia sobre la base de datos de la SRTM-3 del USGS (https://dds.cr.usgs.gov/srtm/).

La variabilidad morfológica de la CARN genera una distribución heterogénea del gradiente pluviométrico Oeste - Este, debido a la presencia de la Cordillera de los Andes que interrumpe el movimiento de las masas de aire del oeste. Las lluvias características en esta región se asocian a sistemas frontales que ingresan desde el Océano Pacífico, desde el sudoeste hacia el noreste. Las precipitaciones (lluvia y nieve) ocurren principalmente en invierno y se asocian a vientos intensos de dirección noroeste, oeste y sudoeste (Bertani, 2011). La precipitación media anual varía entre los 411,7 mm (Estación Rahueco) y 979,9 mm (Estación Buta Mallín), y puede alcanzar una máxima anual de 1.460,0 mm (Estación Buta Mallín). De igual manera, la temperatura se ve afectada por las diferencias topográficas al disminuir con la altura, con un rango entre 24,6 ${ }^{\circ} \mathrm{C}$ y $5,4^{\circ} \mathrm{C}$ a $876,0 \mathrm{~m}$ s.n.m. en el cierre de la cuenca (Estación Rahueco). Su valor medio decrece a medida que se asciende, alcanzando entre $14,8^{\circ} \mathrm{C}$ y $-3,5^{\circ} \mathrm{C}$ a $1.963,0 \mathrm{~m}$ s.n.m. (Estación Buta Mallín) y entre 13,2 ${ }^{\circ} \mathrm{C}$ y $-6,0^{\circ} \mathrm{C}$ a 2.580,0 m s.n.m (Estación Pampa Chacaico) (Figura 2). 
FIGURA 2

Climogramas de las estaciones meteorológicas a) Rahueco

(Ra), b) Buta Mallín (BM) y c) Pampa Chacaico (PC)

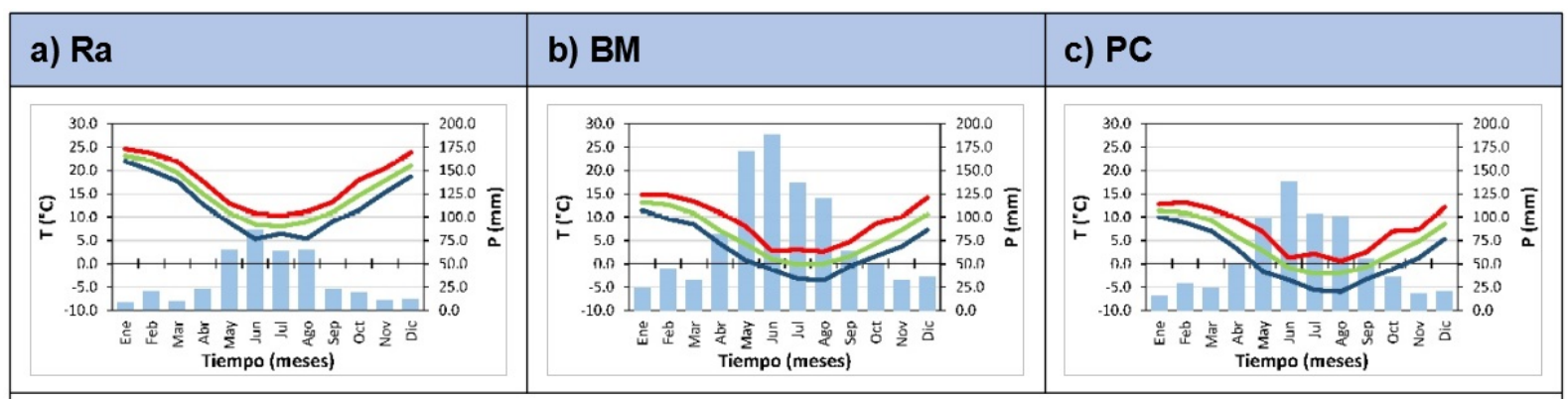

Referencias: La temperatura mensual del aire $\left(T,{ }^{\circ} \mathrm{C}\right)$ se representa mediante líneas de color verde: media; azul: mínima media y roja: máxima media, y la precipitación media mensual $(P, \mathrm{~mm})$, en barras azules.

Fuente: Elaboración propia sobre la base de datos provistos por la Autoridad Interjurisdiccional de las Cuencas de los Ríos Limay, Neuquén y Negro (AIC)

El balance hídrico en Rahueco presenta déficit hídrico en los meses estivales y requerimientos hídricos cubiertos en el invierno, mientras que en las estaciones ubicadas en zonas de cordillera (BM y PC) (Figura 3) las condiciones hídricas son más favorables, con precipitaciones mayores a la evapotranspiración. Estas áreas son muy sensibles a las alteraciones en la disponibilidad del agua, lo que hace que el estudio de la variabilidad asociada al ENOS adquiera suma importancia.

FIGURA 3

Balances hídricos de las estaciones meteorológicas a) Rahueco (Ra), b) Buta Mallín (BM) y c) Pampa Chacaico (PC)

\begin{tabular}{|c|c|c|}
\hline a) $\mathrm{Ra}$ & b) $\mathrm{BM}$ & c) PC \\
\hline 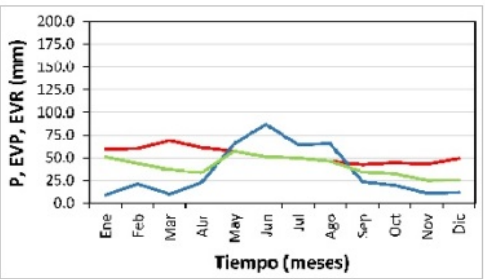 & 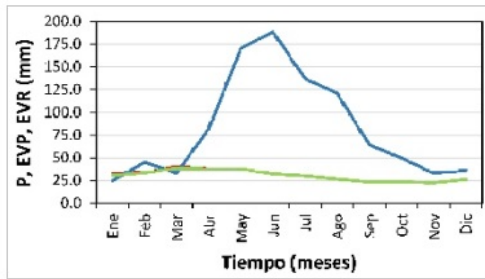 & 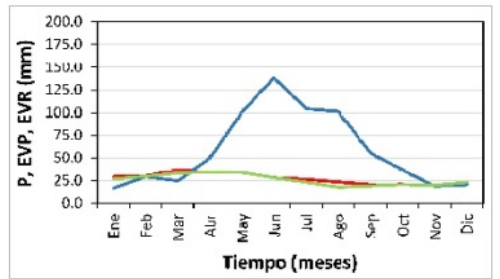 \\
\hline
\end{tabular}

Fuente: Elaboración propia sobre la base de datos provistos por la Autoridad Interjurisdiccional de las Cuencas de los Ríos Limay, Neuquén y Negro (AIC)

La existencia del gradiente altitudinal y las precipitaciones, la orientación de las laderas, las condiciones morfológicas, hidrológicas y el tipo de sustrato condicionan el desarrollo y el tipo de la vegetación natural, observándose a grandes rasgos la provincia fitogeográfica Patagónica hasta los 1.600,0 - 1.800,0 m s.n.m., la Subantártica en algunos sectores entre 1.300,0 - 1.500,0 m s.n.m. y la Altoandina por encima de los 2.800,0 - 3.000,0 m s.n.m. (Cabrera, 1976; Bertani, 2011). 


\section{Metodología}

Se obtuvieron las series de ONI, caudal, precipitación, temperatura, EAN y ACN para el período 2000-2014. Se seleccionó este período de 15 años para unificar la información a partir de la disponibilidad de información satelital, disponible desde el año 2000.

El ONI fue obtenido de la National Oceanic and Atmospheric Administration (NOAA, http:// www.cpc.ncep.noaa.gov/products/analysis_monitoring/ensostuff/ensoyears.shtml), mediante el cual se identificaron las fases cálidas (anomalías positivas, El Niño), frías (anomalías negativas, La Niña) o neutras del fenómeno ENOS al considerar las anomalías de la TSM en la región Niño $3,4\left(5^{\circ} \mathrm{N}-5^{\circ} \mathrm{S}\right.$ y $\left.120^{\circ}-150^{\circ} \mathrm{O}\right)$ en el océano Pacífico Tropical (Figura 4). El índice se calcula a partir de la media móvil de tres meses de las anomalías de la TSM, definiendo fases de El Niño cuando la anomalía supera $+0,5^{\circ} \mathrm{C}$ y de La Niña con anomalías inferiores a $-0,5^{\circ} \mathrm{C}$, en cinco casos consecutivos.

Se analizaron las fases de El Niño y La Niña por separado y ambas en conjunto. Los valores comprendidos entre $-0,5^{\circ} \mathrm{C}$ y $0,5^{\circ} \mathrm{C}$ se asociaron a una fase neutral y fueron considerados como una fase diferente. La intensidad de las fases se consideró mediante el análisis de anomalías de la TSM, definiendo eventos débiles con anomalías entre $\pm 0,5^{\circ} \mathrm{C}$ y $\pm 0,9^{\circ} \mathrm{C}$, moderados entre $\pm 1,0{ }^{\circ} \mathrm{C}$ y $\pm 1,4^{\circ} \mathrm{C}$, fuertes entre $\pm 1,5^{\circ} \mathrm{C}$ y $\pm 1,9^{\circ} \mathrm{C}$ y muy fuertes como mayores/menores a $\pm 2,0^{\circ} \mathrm{C}$.

\section{FIGURA 4}

Anomalías del índice ONI $\left({ }^{\circ} \mathrm{C}\right)$ en la región Niño 3,4 para el período 2000-2014

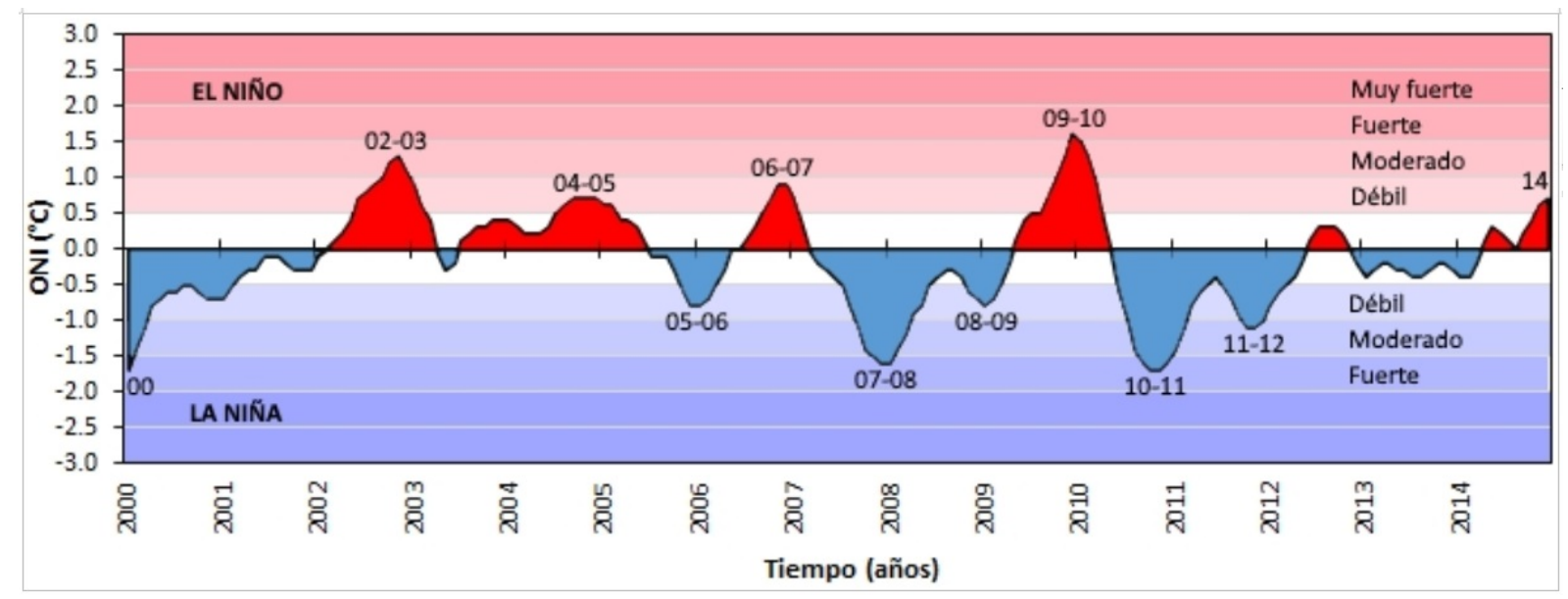

Fuente: Elaboración propia sobre la base de información proporcionada por la NOAA (http://www.cpc.ncep.noaa.gov/products/analysis_monitoring/ensostuff/ensoyears.shtml).

Las series de variables hidrometeorológicas fueron proporcionadas por la Autoridad Interjurisdiccional de las Cuencas de los Ríos Limay, Neuquén y Negro (AIC), siendo analizadas la temperatura media mensual del aire ( $\left.\mathrm{T}-{ }^{\circ} \mathrm{C}-\right)$, la precipitación media mensual ( $\left.\mathrm{P}-\mathrm{mm}-\right)$, el caudal medio mensual $\left(\mathrm{C}-\mathrm{m}^{3} / \mathrm{s}-\right)$ y el Equivalente de Agua en Nieve medio mensual (EAN -mm-). Las estaciones de superficie utilizadas fueron: Rahueco (Ra), localizada al cierre de la cuenca $\left(37^{\circ} 21^{\prime} 20,5^{\prime \prime}\right.$ S, 70²7'11,8" O, 876,0 m s.n.m.); Buta Mallín (BM),

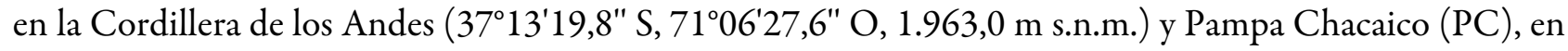
la Cordillera del Viento (36 $28^{\prime} 56,8^{\prime \prime} \mathrm{S}, 70^{\circ} 30^{\prime} 10,1^{\prime \prime O}, 2.580,0 \mathrm{~m}$ s.n.m.) (Figura 1). Las estaciones fueron seleccionadas por su distribución espacial dentro de la CARN y por la confiabilidad que presentan sus datos. Las series de datos originales fueron sometidas a un control de calidad a partir de la observación de valores excepcionales (o extremos) y valores faltantes, sin encontrarse anomalías en ninguno de los casos.

La información satelital incluyó datos de Área Cubierta de Nieve (ACN, km²) (Hall, Salomonson \& Riggs, 2006) y de la National Aeronautics and Space Administration (NASA, https://search.earthdata.nasa.gov), 
y el ACN se generó a partir de 679 escenas MOD10A2, obtenidas por el satélite Terra-MODIS entre el 26/02/2000 al 31/12/2014. Para el trabajo se utilizó el subproducto Maximum Snow Extent, con corrección radiométrica y geométrica (Nivel 3, Colección 5) y una resolución espacial de 500,0 m; la información satelital se procesó mediante ENVI 5.0 (ENvironment for Visualizing Images). Debido a que el subproducto se obtiene cada ocho días de ACN, las escenas fueron ajustadas a una escala media mensual para su adecuación con el índice ONI y variables hidrometeorológicas.

Cabe destacar que las variables temperatura y precipitación fueron empleadas para la caracterización del área de estudio, donde se realizaron climogramas y balances hídricos de las estaciones consideradas. Estos últimos fueron de utilidad para definir los déficits y excesos de agua, y fueron obtenidos a partir de lo propuesto por Thornthwaite (1948) mediante el modelo desarrollado por McCabe y Markstrom (2007) para la USGS. El modelo incluye la nieve en el cálculo, por lo que se ajusta al hecho de que en la CARN la cobertura nívea puede alcanzar la totalidad del área en algunos años (98,2\% en 2007 y 96,6\% en 2011) (Finessi y Groch, 2018).

El análisis de todas las series de datos incluyó el cálculo de correlaciones y significación mediante pruebas de Spearman, para series con distribución no normal, mediante la herramienta XLSTAT, considerando las distintas fases del fenómeno ENOS.

Se obtuvieron además los valores medios, los desvíos estándar y los coeficientes de correlación $(r)$ para la estimación de la relación lineal entre dos variables, considerando un nivel de significación del 5\% (Anderson, Sweeney \& Williams, 2008).

Las correlaciones de temperatura y precipitación fueron realizadas para las estaciones Ra, BM y PC; el análisis de EAN se efectuó para las estaciones de cordillera (BM y PC); el del caudal del río Neuquén se efectuó para la estación Ra, punto de cierre de la cuenca, mientras que el ACN incluyó la totalidad de la cuenca. La intensidad de las asociaciones fue definida por rangos como muy débiles ( $r$ entre 0,00 y $\pm 0,19)$, débiles ( $r$ entre $\pm 0,20 \mathrm{y} \pm 0,39)$, moderadas ( $r$ entre $\pm 0,40$ y $\pm 0,59)$, fuertes ( $r$ entre $\pm 0,60$ y $\pm 0,79$ ) y muy fuertes ( $r$ entre $\pm 0,80$ y $\pm 1,00$ ).

Se efectuaron pruebas de hipótesis, considerando como hipótesis $\left(\mathrm{H}_{0}\right)$ que no es significativo y como hipótesis alternativa $\left(\mathrm{H}_{1}\right)$ que $r$ es significativo. Se plantea aceptar $\mathrm{H}_{1}$ si los coeficientes son significativos al $5 \%$, considerando para ello que la distribución de los datos no es normal.

\section{RESULTADOS Y DISCUSIÓN}

En el período 2000-2014 se identificaron once eventos El Niño - Oscilación del Sur (ENOS) (Figura 4) y seis eventos de intensidad moderada y fuerte siguiendo los criterios de la NOAA. Se clasificaron como El Niño los ocurridos en 2002-2003 y 2009-2010, y como La Niña, los ocurridos en 2000, 2007-2008, 2010-2011 y 2011-2012. A partir de dicha identificación, se analizaron los cambios en la temperatura (T), precipitación (P), Equivalente de Agua en Nieve (EAN), Área Cubierta de Nieve (ACN) y caudal (C), con variaciones espaciales, altitudinales y temporales y su asociación con ENOS.

Las estaciones analizadas presentan comportamientos asociados a una menor disponibilidad de agua en época estival, siendo máximo en diciembre - enero. En ese aspecto se analizó si los años asociados a El Niño o La Niña presentaron variaciones estadísticamente significativas en las variables.

Durante las fases de El Niño en 2000-2014 (Figura 4), la temperatura en las estaciones de altura (BM y PC) fue menor a la media mensual del período $\left(-0,5^{\circ} \mathrm{C}\right.$ en $\mathrm{BM}$ y $-0,7^{\circ} \mathrm{C}$ en PC) y el Área Cubierta de Nieve (ACN) presentó una anomalía positiva del 10,6\% durante la fase El Niño y una anomalía negativa de 6,6\% durante la fase La Niña (Tabla 1). Este efecto se observó también en el Equivalente de Agua en Nieve, ya que en las estaciones de altura el EAN presentó anomalías positivas de 10,8\% en BM y 23,6\% en PC durante las fases El Niño y anomalías negativas de 7,7\% en BM y de 16,8\% en PC en las fases La Niña (Tabla 1). Sin embargo, la estación $\mathrm{Ra}$, a menor altura, exhibió un comportamiento inverso, con un leve descenso de 
la temperatura $\left(\approx-0,3^{\circ} \mathrm{C}\right)$ y de la precipitación $(\approx-3,9 \mathrm{~mm})$, detectado como un leve incremento del caudal $\left(\approx 47,9 \mathrm{~m}^{3} / \mathrm{s}\right)$ del río Neuquén. En esta estación, los valores máximos de las variables consideradas en la fase La Niña coincidieron con los máximos del período estudiado (Tabla 1).

El caudal medio del río Neuquén del período $2000-2014$ fue de $224,3 \mathrm{~m}^{3} / \mathrm{s}$, con un máximo de $610,0 \mathrm{~m}^{3} /$ s y un valor mínimo de $13,7 \mathrm{~m}^{3} / \mathrm{s}$, ambos en fase débil de La Niña.

TABLA 1

Estadísticas descriptivas para el período 2000-2014 discriminando en los períodos con ocurrencia de El Niño y La Niña

\begin{tabular}{|c|c|c|c|c|c|c|c|c|c|c|c|}
\hline \multicolumn{2}{|c|}{ Estación } & \multicolumn{3}{|c|}{$\mathrm{Ra}$} & \multicolumn{3}{|c|}{$\overline{B M}$} & \multicolumn{3}{|c|}{$\overline{P C}$} & \multirow{2}{*}{$\begin{array}{c}\begin{array}{c}\text { Total } \\
\text { cuenca }\end{array} \\
\text { ACN } \\
\left(\mathrm{km}^{2}\right)\end{array}$} \\
\hline & & $\underset{(\mathrm{mm})}{P}$ & $\begin{array}{c}\mathrm{T} \\
\left({ }^{\circ} \mathrm{C}\right)\end{array}$ & $\underset{\left(m^{3} / s\right)}{C}$ & $\underset{(\mathrm{mm})}{P}$ & $\begin{array}{c}\mathrm{T} \\
\left({ }^{\circ} \mathrm{C}\right)\end{array}$ & $\begin{array}{l}\text { EAN } \\
(\mathrm{mm})\end{array}$ & $\underset{(\mathrm{mm})}{P}$ & $\begin{array}{c}\mathrm{T} \\
\left({ }^{\circ} \mathrm{C}\right)\end{array}$ & $\begin{array}{l}\text { EAN } \\
(\mathrm{mm})\end{array}$ & \\
\hline \multirow{4}{*}{$\begin{array}{l}\text { Periodo } \\
\text { completo } \\
2000- \\
2014\end{array}$} & $x$ & 0,0 & 5,3 & 13,7 & 0,0 & $-3,5$ & $-0,2$ & 0,0 & $-6,0$ & 0,0 & 7,5 \\
\hline & $\overline{X_{m}}$ & 324,0 & 24,6 & 610,0 & 551,0 & 14,7 & $1.122,2$ & 377,0 & 13,2 & $1.959,9$ & 7277,5 \\
\hline & म & 29,8 & 16,2 & 224,3 & 77,9 & 6,7 & 278,3 & 55,4 & 4,7 & 513,1 & $1.989,2$ \\
\hline & $\overrightarrow{0}$ & 45,8 & 5,7 & 150,6 & 90,7 & 5,3 & 347,1 & 64,7 & 5,5 & 555,9 & $2.224,7$ \\
\hline \multirow[t]{4}{*}{ El Niño } & $\bar{X}$ & 0,0 & 5,3 & 39,4 & 0,0 & $-0,6$ & $-0,2$ & 0,0 & $-3,5$ & 0,0 & 17,9 \\
\hline & $x$ & 172,0 & 23,6 & 567,1 & 284,0 & 14,7 & $1.113,8$ & 246,0 & 13,2 & $1.815,1$ & $6.055,0$ \\
\hline & म & 25,9 & 15,9 & 272,2 & 83,4 & 6,2 & 308,3 & 61,9 & 4,0 & 634,0 & 2200,3 \\
\hline & 局 & 35,6 & 5,4 & 138,3 & 71,3 & 4,9 & 359,1 & 54,3 & 5,2 & 600,0 & $2.189,9$ \\
\hline \multirow{4}{*}{ La Niña } & $\overline{X_{m}}$ & 0,0 & 5,4 & 13,7 & 0,0 & $-3,5$ & 0,0 & 0,0 & $-6,0$ & 0,0 & 7,5 \\
\hline & $x$ & 324,0 & 24,6 & 610,0 & 551,0 & 14,7 & $1.122,2$ & 377,0 & 13,2 & $1.959,9$ & $7.277,5$ \\
\hline & $\mu$ & 32,3 & 16,4 & 194,9 & 74,5 & 7,0 & 256,9 & 51,4 & 5,1 & 427,1 & $1.857,2$ \\
\hline & 0 & 51,3 & 5,9 & 151,5 & 101,2 & 5,6 & 340,8 & 70,5 & 5,6 & 511,8 & $2.255,7$ \\
\hline \multicolumn{12}{|c|}{$\begin{array}{l}\text { Referencias: Variables P }(\mathrm{mm}) \text { : precipitación media mensual; } \mathrm{T}\left({ }^{\circ} \mathrm{C}\right) \text { : temperatura media mensual del aire; C } \\
\left(\mathrm{m}^{3} / \mathrm{s}\right) \text { : caudal medio mensual; EAN (mm): Equivalente de Agua en Nieve medio mensual; } \mathrm{ACN}\left(\mathrm{km}^{2}\right) \text { : Área } \\
\text { Cubierta de Nieve media mensual; estadisticas } x_{\operatorname{mir}} \text { : valor minimo; } x_{\max } \text { : valor máximo; } \mu \text { : valor medio de la } \\
\text { población; y } \sigma \text { : desvío estándar. }\end{array}$} \\
\hline
\end{tabular}

Fuente: Elaboración propia sobre la base de datos de la AIC, NOAA y NASA

La Figura 5 presenta las relaciones entre las distintas variables estudiadas y el ONI en la CARN. Los diagramas de dispersión muestran una débil asociación, con correlaciones medias de 0,35 al 5\%.

La precipitación en las tres estaciones no presenta una asociación marcada con ninguna de las fases; sin embargo, en BM y en PC la correlación con La Niña es significativa con $r=0,44$ y 0,30 , respectivamente; asimismo, en $\mathrm{Ra}$ este comportamiento es similar con $r=0,25$, sin ser significativo. Estos valores insinuarían una relación entre La Niña y la precipitación. La asociación entre la temperatura y el ONI presenta una mayor dispersión que la precipitación, insinuando una correlación positiva con El Niño y negativa con La Niña, sin llegar a ser significativa en ninguno de los casos. 
FIGURA 5

Valores medios del coeficiente de correlación $(r)$ entre $\mathrm{P}(\mathrm{mm}), \mathrm{T}\left({ }^{\circ} \mathrm{C}\right), \mathrm{EAN}(\mathrm{mm}), \mathrm{ACN}$ $\left(\mathrm{km}^{2}\right), \mathrm{C}\left(\mathrm{m}^{3} / \mathrm{s}\right)$ y ONI $\left({ }^{\circ} \mathrm{C}\right)$ en el período 2000-2014, para las estaciones Ra, BM y PC

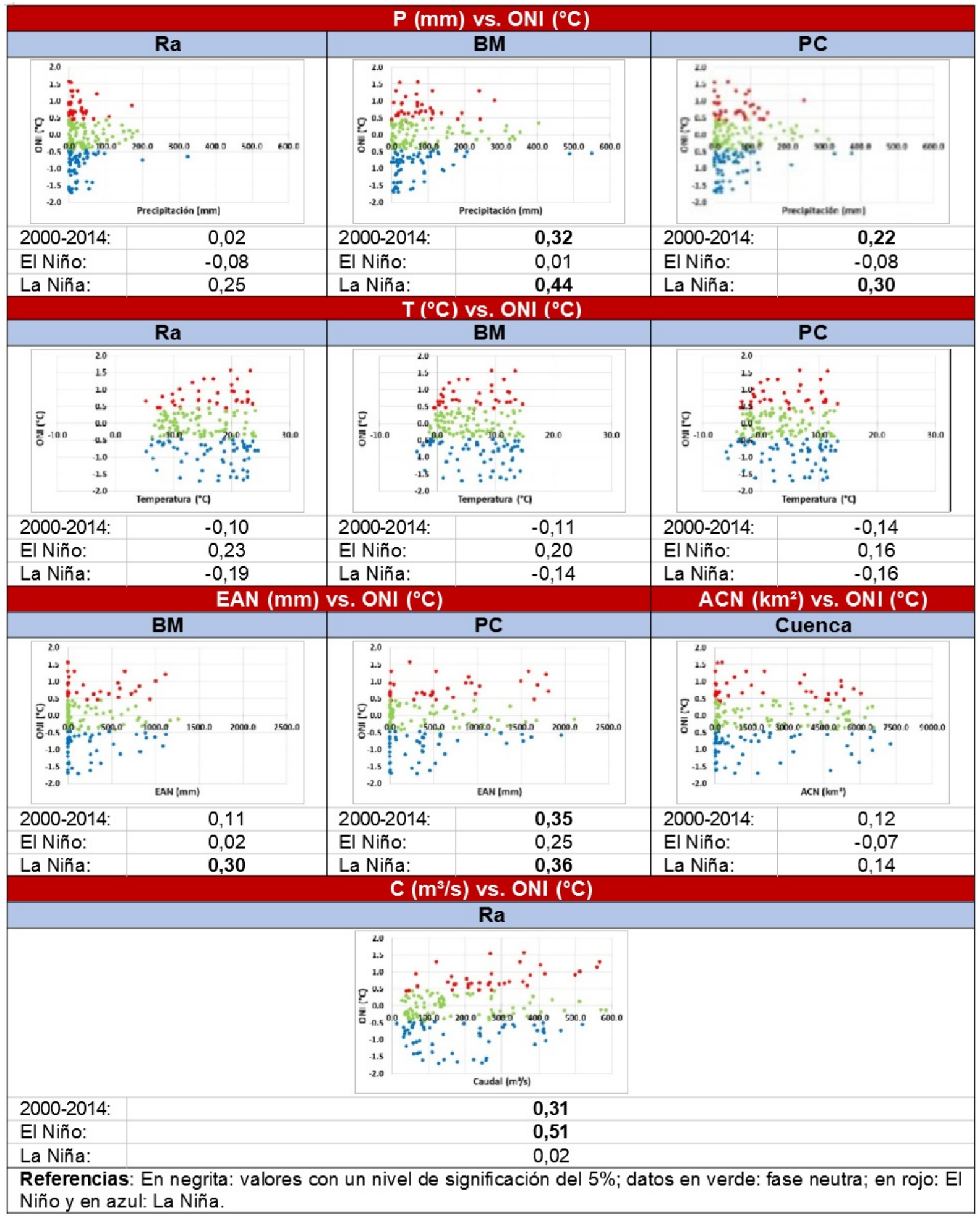

La correlación entre el EAN y el ONI tuvo un comportamiento similar a la precipitación en BM y en PC, presentando una asociación significativa con La Niña con $r=0,30$ y 0,36, respectivamente. El ACN presentó una correlación muy débil, entre - 0,07 y 0,14, negativa para El Niño y positiva para La Niña. Estos resultados 
presentan concordancia con los de Escobar y Aceituno (1998), quienes observaron una menor influencia de los fenómenos El Niño y La Niña sobre la acumulación de nieve al sur de los $35^{\circ} \mathrm{S}$ en el sector chileno de la Cordillera de los Andes para 1951-1997.

La correlación entre el caudal y el ONI fue de 0,51 para la totalidad del período y 0,31 para las fases de El Niño, con un nivel de significación del 5\%, mientras que fue de 0,02 para las fases de La Niña, indicando correlaciones moderadas a débiles y muy débiles, respectivamente, en el área de estudio. Estos resultados fueron similares a los encontrados por Compagnucci y Araneo $(2005,2007)$ para los ríos andinos argentinos, utilizando datos de estaciones meteorológicas para registros que engloban distintos períodos entre 1903 y 2004.

Teniendo en cuenta las correlaciones entre la temperatura del aire y caudal con los eventos ENOS, la temperatura en Ra a menor altitud presentó una máxima correlación negativa con el ONI $(r=-0,19)$ durante la fase La Niña y un máximo positivo en esa estación (Ra) entre el caudal y el ONI de 0,51 durante la fase El Niño. Sin embargo, aun cuando en el período los extremos de caudal se dan en fases frías, este no presenta una asociación estadísticamente significativa con La Niña $(r=0,02)$ (Figura 5), lo que requeriría el análisis de series de datos más extensas.

\section{Conclusiones}

Las variables analizadas en este trabajo sugieren correlaciones débiles y moderadas, sin un comportamiento definido para las distintas fases de ENOS. La precipitación, la temperatura, el caudal, el Equivalente de Agua en Nieve y el Área Cubierta de Nieve presentaron una débil asociación con el ONI, con un coeficiente de correlación medio de 0,35 y un nivel de significación del 5\% en la Cuenca Alta del Río Neuquén (CARN) para el período 2000-2014.

Durante la fase La Niña la temperatura en Ra presentó una máxima correlación inversa con el ONI de $-0,19$ y un máximo positivo entre el caudal y el ONI en Ra de 0,51 durante la fase El Niño.

El ACN fue un 10,6\% mayor a la media del período total durante la fase El Niño y un 6,6\% menor durante la fase La Niña. En las estaciones de altura el EAN fue un 10,8\% mayor en BM y un 23,6\% en PC durante las fases El Niño y durante la Niña fue 7,7\% menor en BM y 16,8\% en PC. La estación Ra exhibió un comportamiento inverso a las estaciones de altura, con un leve descenso de la temperatura $\left(\approx-0,3{ }^{\circ} \mathrm{C}\right)$ y la precipitación $(\approx-3,9 \mathrm{~mm})$, y un leve incremento del caudal $\left(\approx 47,9 \mathrm{~m}^{3} / \mathrm{s}\right)$. Los valores máximos de las variables consideradas en la fase La Niña coincidieron con los máximos del período estudiado.

Los resultados obtenidos en el presente estudio concuerdan con los llevados a cabo en similares sectores andinos por Escobar y Aceituno (1998) para la precipitación nívea y por Compagnucci y Araneo (2005, 2007) para el caudal.

El área en estudio incluye sistemas naturales sensibles a la disponibilidad hídrica y, además, teniendo en cuenta que las variaciones existentes en la frecuencia e intensidad de precipitación se asocian con eventos que afectan a la población, como inundaciones, sequías y grandes nevadas, un mayor conocimiento de las relaciones existentes sería importante para la planificación de estrategias de manejo del agua y para la preparación de alternativas de mitigación de los problemas asociados a ello en el sector socioproductivo y energético en la cuenca, y en las aguas debajo de ella.

\section{Agradecimientos}

Se agradecen los datos provistos por la Autoridad Interjurisdiccional de las Cuencas de los Ríos Limay, Neuquén y Negro, información sin la cual no hubiese sido posible la realización del presente trabajo. 


\section{REFERENCIAS}

Aceituno, P. (1990). Variabilidad interanual en el caudal de ríos andinos en Chile Central en relación con la temperatura de la superficie del mar en el Pacífico central. Revista de la Sociedad Chilena de Ingeniería Hidráulica, 5, 7-19.

Anderson, D. R., Sweeney, D. J. y Williams, T. A. (2008). Estadística para administración y economia. México D.F.: Cengage Learning Editores.

Araya Ojeda M. e Isla F. I. (2016). Variabilidad hidrológica en la región del Biobío: los eventos El Niño en zonas templadas de Chile. Revista Universitaria de Geografia, 25, 31-55.

Bertani, L. A. (2011). Evaluación geoecológica de los paisajes del Departamento Minas (Provincia de Neuquén), para el estudio de la degradación de la tierra. Tesis de Doctorado. Universidad Nacional de La Plata. Facultad de Humanidades y Ciencias de la Educación. La Plata.

Cabrera, A. L. (1976). Regiones Fitogeográficas Argentinas. Buenos Aires: ACME.

Callau Poduje, A. C. \& Seoane, R. (2013). Effect of ENSO-based climate variability in the estimation of flood events in Argentina. En Jensen, J. (ed.), Proceedings of the 1st International Short Conference on Advances in Extreme Value Analysis and Application to Natural Hazards (pp. 124-136). Siegen, Alemania. Recuperado de: https://w ww.uni-siegen.de/evan2013/home/proceedings/evan2013_proceedings.pdf

Cerveny, R., Skeeter, B. \& Dewey, K. (1987). A preliminary investigation of a relationship between South American snow cover and the Southern Oscillation. Monthly Weather Review. 115, 620-623. doi: https://10.1175/1520-0493(1987)115<0620:APIOAR>2.0.CO;2.

CIMOP (2009). La gestión del agua y su infraestructura en el desarrollo sustentable del territorio. Recuperado de: $\mathrm{h}$ ttp://www.cimop.gov.ar/gestion_agua.rar

Compagnucci, R. H. y Araneo, D. C. (2005). Identificación de áreas de homogeneidad estadística para los caudales de ríos andinos argentinos y su relación con la circulación atmosférica y la temperatura superficial del mar. Meteorológica, 30, 41-53.

Compagnucci, R. H. y Araneo, D. C. (2007). Alcances de El Niño como predictor del caudal de los ríos andinos argentinos. Ingeniería Hidráulica en México, 22, 23-35.

Compagnucci, R. H. \& Vargas, W. M. (1998). Inter-annual variability of Cuyo Rivers Streamflow in Argentinean Andean Mountains and ENSO events. International Journal of Climatology, 18, 1593-1609. doi: https://10.1002/(SICI)1097-0088(19981130)18:14<1593::AID-JOC327>3.0.CO;2-U.

Daniels, L. D. \& Veblen, T. T. (2000). ENSO effects on temperature and precipitation of the Patagonian-Andean region: implications for biogeography. Physical Geography, 3, 223-243.

Escobar, F. y Aceituno, P. (1998). Influencia del fenómeno ENSO sobre la precipitación nival en el sector andino de Chile Central durante el invierno. Bulletin de l'Institut français d'études andines, V (27), 753-759.

Finessi, G. F. y Groch, D. (2018). Estudio hidrológico de la Cuenca Alta del Río Neuquén. Tesis de Licenciatura. Universidad Nacional del Comahue, Facultad de Humanidades. Neuquén. doi: https://10.13140/RG.2.2.187 95.90403.

García, N. O. (1994). South american climatology. Quaternary International, 21, 7-27. doi: https://10.1016/1040-6 182(94)90018-3.

Gómez Cole, M. (2010). Variaciones de la cubierta de nieve en Chile centro - sur $\left(30^{\circ}-41^{\circ}\right.$ s $)$ y su relación con el fenómeno ENSO. Tesis de Grado. Universidad de Concepción, Facultad de Arquitectura, Urbanismo y Geografía. Concepción.

Hall, D. K., Salomonson, V. V. \& Riggs, G. A. (2006). MODIS/Terra Snow Cover 8-Day L3 Global 500m Grid. Version 5. [MOD10A2]. NASA National Snow and Ice Data Center Distributed Active Archive Center. doi: https://10.5067/ACYTYZB9BEOS.

Lauro, C., Vich, A. y Moreiras, S. M. (2016). Variabilidad del régimen fluvial en cuencas de la región de Cuyo. GEOACTA, 40, 28-51. 
Martínez, C., Fernández, A. y Rubio, P. (2012). Caudales y variabilidad climática en una cuenca de latitudes medias en Sudamérica: río Aconcagua, Chile Central (33ㅇ). Boletín de la Asociación de Geógrafos Españoles. 58, 227-248.

Masiokas, M., Villalba, R., Luckman, B. H., Le Quesne, C. \& Aravena, J. (2006). Snowpack variations in the Central Andes of Argentina and Chile, 1951-2005: Large-Scale Atmospheric Influences and Implications for Water Resources in the Region. Journal of Climate, 19, 6334-6352. doi: https://10.1175/JCLI3969.1.

Masiokas, M. H., Villalba R., Christie, D. A., Betman, E., Luckman, B. H., Le Quesne, C., Prieto, M. R. \& Mauget, S. (2012). Snowpack variations since AD 1150 in the Andes of Chile and Argentina $\left(30^{\circ}-37^{\circ} \mathrm{S}\right)$ inferred from rainfall, tree-ring and documentary records. Geophysical Research. 117, 1-11. doi: https://10.1029/2011JD01 6748.

McCabe, G.J. \& Markstrom, S.L. (2007). A monthly water-balance model driven by a graphical user interface. U.S. Geological Survey. doi: https://10.3133/ofr20071088.

NASA (2019). MOD10A2 collection. https://search.earthdata.nasa.gov/search [consulta: 06/01/2019].

NOAA (2016). El Niño / Southern Oscillation (ENSO): Cold \& Warm Episodes by Season. http://www.cpc.ncep. noaa.gov/products/analysis_monitoring/ensostuff/ensoyears.shtml [consulta: 08/09/2016].

Norte, F., Simonelli, S. y Heredia, N. (1998). Impacto del fenómeno ENOS en el régimen hidrometeorológico de Mendoza, Argentina. Bulletin de l'Institut français d'etudes andines, V (27), 761-770.

Penalba, O.C. \& Ribera, J.A. (2016). Precipitation response to El Niño/La Niña events in Southern South America - emphasis in regional drought occurrences. Advances in Geosciences. 42, 1-14. doi: https://10.5194/adgeo-42 $-1-2016$.

Ribera, J. A. \& Penalba, O. C (2015). El Niño/La Niña events as a tool for regional drought monitoring in Southern South America. En J. Andreu, A. Solera, J. Paredes-Arquiola, D. Haro-Monteagudo \& H. van Lanen (Eds.). Drought: Research and Science-Policy Interfacing (pp. 293-299). Londres: CRC Press.

Ropelewski, C. F. \& Halpert, M.S. (1989). Precipitation patterns associated with the high index phase of the Southern Oscillation. Journal of Climate, 2, 268-284. doi: https://10.1175/1520-0442(1989)002<0268:PPAWTH>2.0.CO;2.

Ropelewski, C. F. \& Halpert, M.S. (1996). Quantifying Southern Oscillation-precipitation relationships. Journal of Climate, 9, 1043-1059. doi: https://10.1175/1520-0442(1996)009<1043:QSOPR>2.0.CO;2.

Ropelewski, C. F. \& Jones, P. D. (1987). An extension of the Tahiti-Darwin Southern Oscillation Index. Monthly Weather Review. 115, 2161-2165. doi: https://10.1175/1520-0493(1987)115<2161:AEOTTS>2.0.CO;2.

Thornthwaite, C.W., (1948). An Approach toward a Rational Classification of Climate. Geographical Review. 38, 55-94. doi: https://10.2307/210739.

Trenberth, K. E. \& Stepaniak, D. P. (2001). Indices of El Niño Evolution. Journal of Climate. 14, 1697-1701. doi: https://10.1175/1520-0442(2001)014<1697:LIOENO>2.0.CO;2.

USGS (2009). Shuttle Radar Topography Mission: Version 2.1. https://dds.cr.usgs.gov/srtm/ [consulta: 25/07/2009].

\section{BY-NC-SA}

